\title{
Waiting time distributions in a two-level fluctuator coupled to a superconducting charge detector
}

\author{
Máté Jenei $\odot,{ }^{1, *}$ Elina Potanina $\odot,{ }^{1, *}$ Ruichen Zhao, ${ }^{2, \dagger}$ Kuan Y. Tan, ${ }^{1}$ Alessandro Rossi $\odot,{ }^{3,4}$ Tuomo Tanttu, ${ }^{2}$ \\ Kok W. Chan $\odot,{ }^{2}$ Vasilii Sevriuk $\odot,{ }^{1}$ Mikko Möttönen, ${ }^{1,5}$ and Andrew Dzurak $\odot^{2}$ \\ ${ }^{1}$ QTF Centre of Excellence, Department of Applied Physics, Aalto University, P.O. Box 13500, FI-00076, Aalto, Finland \\ ${ }^{2}$ School of Electrical Engineering and Telecommunications, University of New South Wales, Sydney, New South Wales 2052, Australia \\ ${ }^{3}$ Department of Physics, SUPA, University of Strathclyde, Glasgow G4 ONG, United Kingdom \\ ${ }^{4}$ National Physical Laboratory, Hampton Road, Teddington TW11 OLW, United Kingdom \\ ${ }^{5}$ VTT Technical Research Centre of Finland Ltd., P.O. Box 1000, FI-02044, VTT, Finland
}

(Received 13 September 2019; revised manuscript received 29 October 2019; published 10 December 2019)

\begin{abstract}
We analyze charge fluctuations in a parasitic state strongly coupled to a superconducting Josephson-junctionbased charge detector. The charge dynamics of the state resembles that of electron transport in a quantum dot with two charge states, and hence we refer to it as a two-level fluctuator. By constructing the distribution of waiting times from the measured detector signal and comparing it with a waiting time theory, we extract the electron in- and out-tunneling rates for the two-level fluctuator, which are severely asymmetric.
\end{abstract}

DOI: 10.1103/PhysRevResearch.1.033163

\section{INTRODUCTION}

Parasitic states including charge traps are present in almost all solid-state devices and there have been several proposals on how to avoid them [1-9]. Two-level fluctuators (TLFs), for example, substantially affect qubit coherence time [10-13] and degrade charge sensing [14,15]. However, if the time scales of charge fluctuations in a trap are significantly different from those of the operation of the actual device, their harmful effect can be mitigated. In silicon, TLFs have been characterized by various approaches using metallic singleelectron transistors [8,16-19], a scheme to which we contribute in this paper.

Electron waiting times have been investigated for a wide range of physical systems including quantum dots [20-36], coherent conductors $[37,38]$, molecular junctions $[39,40]$, and superconducting systems [41-47]. Distributions of waiting times contain complementary information on charge transport properties which is not necessarily encoded in the full counting statistics (FCS) and vice versa [20]. For example, waiting time distributions capture the interference effects in double-dot setups [21], reveal the correlations in multichannel systems $[25,48]$, allow us to separate slow and fast dynamics in Cooper-pair splitters [46], resolve few-photon processes [49], and even investigate the topological superconductivity in hybrid junctions $[45,47]$. In dynamic, periodically driven systems, waiting time distributions are clear indicators of regular single-electron transport [50-53]. Furthermore, waiting

\footnotetext{
*These authors contributed equally to this work.

${ }^{\dagger}$ Present address: NIST 325 Broadway, Boulder, CO 80305, USA.
}

Published by the American Physical Society under the terms of the Creative Commons Attribution 4.0 International license. Further distribution of this work must maintain attribution to the author(s) and the published article's title, journal citation, and DOI. time distributions were used in a recent experiment [54] to optimize single-electron spin-readout fidelity.

In this work, we investigate electronic waiting times between charge transitions in and out of a parasitic state to (a)

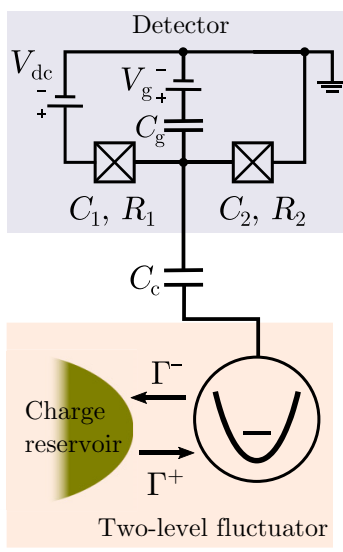

(b)

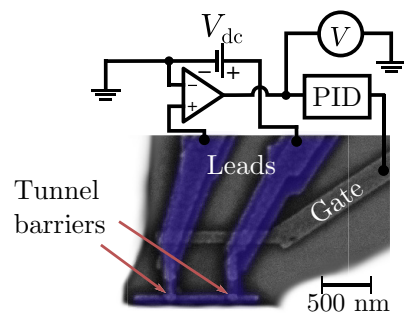

(c)

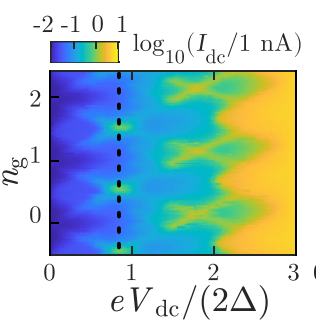

(d) Data - Lorenzian - Sensitivity

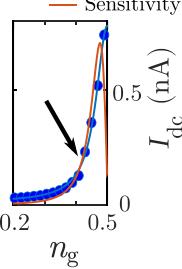

FIG. 1. (a) Schematic diagram of the investigated system which consists of a Josephson-junction-based detector (top) and a two-level system (bottom) that is in tunnel contact with a charge reservoir. All symbols are described in the main text. (b) False-color scanning electron micrograph of the charge detector similar to that in the measurements together with a circuit diagram of the experimental setup. (c) Detector current as a function of the normalized bias voltage $e V_{\mathrm{dc}} /(2 \Delta)$ and the gate charge $n_{\mathrm{g}}=V_{\mathrm{g}} C_{\mathrm{g}} / e$, where $\Delta$ is the superconducting gap. The dashed line indicates the bias point for the measurement, which (d) is plotted along, where a Lorentzian function (blue line) is fitted to the data (blue circle) that is used to estimate the sensitivity (red) in arbitrary units. The black arrow indicates the PID set point. The parameters of the measured device are $\Delta=195 \mu \mathrm{eV}, R_{\mathrm{T}}=R_{1}+R_{2}=180 \mathrm{k} \Omega$, and $E_{\mathrm{ch}}=160 \mu \mathrm{eV}$ 
directly extract the time scales of such TLF. We employ a superconducting single-electron transistor (SSET) to monitor the switching events on the TLF and apply a continuous electrostatic feedback on the detector to maintain a constant charge sensitivity, which tends to fasten an asymmetry in the detected in- and out-tunneling rates.

This paper is organized as follows: In Sec. II, we provide a short overview of the waiting time theory [20] and illustrate these concepts by evaluating the distribution of electron waiting times for a TLF in Secs. II A and II B. We treat the two-level fluctuator as a potential well that is in tunnel contact with a charge reservoir as illustrated in Fig. 1(a). In Sec. III, we discuss our experimental setup. In Sec. IV, we present our measurement results and compare them with the waiting time distribution theory. Since our model assumes sequential in- and out-tunneling, we calculate the FCS of the switching events and compare the results with the waiting times. Finally, in Sec. V we conclude our work.

\section{ELECTRON WAITING TIMES}

The time that passes between two subsequent singleelectron tunneling events of the same type is usually referred to as the electron waiting time $\tau[20,37,50]$. The single-electron tunneling process has a stochastic nature and therefore is described by a waiting time distribution (WTD) function $\mathcal{W}(\tau)$. For stationary transport problems, the WTD relates to the idle-time probability $\Pi(\tau)$ as $[37,38]$

$$
\mathcal{W}(\tau)=\langle\tau\rangle \partial_{\tau}^{2} \Pi(\tau),
$$

where $\Pi(\tau)$ is the probability of having no tunneling events during a time $\operatorname{span} \tau$. The mean waiting time $\langle\tau\rangle$ can be expressed in terms of the idle-time probability as $[37,38]$ $\langle\tau\rangle=\int_{0}^{\infty} d \tau \mathcal{W}(\tau) \tau=-1 / \dot{\Pi}(\tau=0)$.

The statistics of single-electron tunneling events is captured by the probability $P(n, t)$ of having $n$ tunneling events of the chosen type during the time span $\left[t_{0}, t_{0}+t\right]$ [55-57]. However, we only need to know the idle-time probability $\Pi(\tau)=P(n=0, \tau)$ to obtain the WTD. In FCS, the moment generating function

$$
\mathcal{M}(\chi, t)=\sum_{n=0}^{\infty} P(n, t) e^{i n \chi}
$$

provides us with all the moments of $n$ as $\left\langle n^{m}\right\rangle(t)=$ $\left.\partial_{i \chi}^{m} \mathcal{M}(\chi, t)\right|_{i \chi \rightarrow 0}$. From Eq. (2) we observe that $\mathcal{M}(i \infty, t)=$ $P(n=0, t)$ is exactly the idle-time probability. Next, we utilize these concepts by evaluating the WTDs for a two-level fluctuator.

\section{A. Waiting times in a two-level fluctuator}

We describe the parasitic state as a single-electron box consisting of a nanoscale island, the charge dynamics of which is governed by the master equation

$$
\frac{d}{d t} \mathrm{p}(t)=\mathbf{L p}(t)
$$

where the vector $\mathrm{p}(t)=\left[p_{0}(t), p_{1}(t)\right]^{T}$ contains the probabilities $p_{0}(t)$ and $p_{1}(t)$ for the island to be empty or occupied by 1 electron, respectively, and the rate matrix $\mathbf{L}$ describes the transitions between 0 and 1 charge states of the island.

We partition the rate matrix as $\mathbf{L}=\mathbf{L}_{0}+\mathbf{J}_{+}+\mathbf{J}_{-}$with jump operators $\mathbf{J}_{ \pm}$describing charge transfers to and from the island, respectively [58]. We resolve the probability vector $\mathrm{p}(n, t)$ such that it accounts for the number of tunneling events $n$. The $n$-resolved equations of motion, $\frac{d}{d t} \mathrm{p}(n, t)=$ $\mathbf{L}_{0} \mathrm{p}(n, t)+\mathbf{J}_{+} \mathrm{p}(n-1, t)+\mathbf{J}_{-} \mathrm{p}(n+1, t)$, are decoupled by introducing the counting field $\chi$ via the definition $\mathrm{p}(\chi, t) \equiv$ $\sum_{n} \mathrm{p}(n, t) e^{i n \chi}$. We then arrive at a modified master equation for $\mathrm{p}(\chi, t)$

$$
\frac{d}{d t} \mathrm{p}(\chi, t)=\mathbf{L}(\chi) \mathrm{p}(\chi, t) .
$$

For $\chi=0$ in Eq. (4), we recover the original master equation (3). Further on, we focus on the waiting times between the into-the-island tunneling events, and hence set $\mathbf{J}_{+}=0$. In this case, the modified rate matrix $\mathbf{L}(\chi)$ assumes the form

$$
\mathbf{L}(\chi)=\left(\begin{array}{cc}
-\Gamma^{+} & \Gamma^{-} \\
e^{i \chi} \Gamma^{+} & -\Gamma^{-}
\end{array}\right) .
$$

We have included the counting factor $e^{i \chi}$ in the lower offdiagonal element together with $\Gamma^{+}$, corresponding to counting the number of tunneling events into the parasitic state [55-57]. The solution of the modified master equation formally reads

$$
\mathrm{p}(\chi, t)=e^{\mathbf{L}(\chi) t} \mathrm{p}(\chi, 0) .
$$

The idle-time probability follows as $\Pi(\tau)=\sum_{j} p_{j}(i \infty, t)$, where $p_{j}(i \infty, t)$ is an $j$ th component of the vector $\left.\mathrm{p}(\chi, t)\right|_{i \chi \rightarrow i \infty}$. For a given rate matrix (5) the solution is analytic and we are able to evaluate the WTD for a two-level fluctuator using relation (1) as

$$
\mathcal{W}(\tau)=\Gamma^{+} \Gamma^{-} \frac{e^{-\Gamma^{-} \tau}-e^{-\Gamma^{+} \tau}}{\Gamma^{+}-\Gamma^{-}},
$$

where $\tau$ is the waiting time between single-electron subsequent tunneling events from the charge reservoir to the parasitic state. Counting the out-of-the-island tunneling events yields an identical result for the distribution of waiting times. We continue with another example of WTD, where we take into account the effect of finite detection time.

\section{B. Residence times in a two-level fluctuator coupled to a detector}

Let us focus on the time that the electron spends in the parasitic state known as the residence time $\tau^{*}$ [59]. We use the term residence time to avoid confusion with the waiting time discussed in the previous section. When the residence time becomes comparable to the inverse of the detector bandwidth, $2 \pi / \Gamma^{\mathrm{D}}$, we have to take it into account when evaluating the distribution of residence times.

Due to the finite bandwidth of the detector, we may fail to detect some of the tunneling events. Therefore, we add two more possible states when formulating the rate equation [60]. The revised probability vector reads $\mathrm{p}^{*}(t)=\left[p_{00}(t), p_{10}(t), p_{01}(t), p_{11}(t)\right]^{T}$. Probabilities $p_{01}(t)$ and $p_{10}(t)$ correspond to the situation when the parasitic state is empty or occupied, respectively, but it has not been detected. The detected empty and occupied states are denoted by $p_{00}(t)$ and $p_{11}(t)$, respectively. 
While actual charge transitions between the occupied state $p_{11}(t)$ to the undetected empty state $p_{01}(t)$ may occur several times before the empty state $p_{00}(t)$ gets detected, we obtain the experimentally observed residence time distribution by counting the events $p_{01}(t) \rightarrow p_{00}(t)$. After including the effect of the finite detector bandwidth $\Gamma^{\mathrm{D}} /(2 \pi)$ in the rate equation, we arrive at the following rate matrix $[61,62]$ :

$$
\mathbf{L}(\chi)=\left(\begin{array}{cccc}
-\Gamma^{+} & \Gamma^{-} & \Gamma^{\mathrm{D}} e^{i \chi} & 0 \\
\Gamma^{+} & -\left(\Gamma^{-}+\Gamma^{\mathrm{D}}\right) & 0 & 0 \\
0 & 0 & -\left(\Gamma^{+}+\Gamma^{\mathrm{D}}\right) & \Gamma^{-} \\
0 & \Gamma^{\mathrm{D}} & \Gamma^{+} & -\Gamma^{-}
\end{array}\right) .
$$

In analogy to the previous section, we solve the modified rate equation given the rate matrix Eq. (8) in the limit $\chi \rightarrow$ $i \infty$ with an initial condition $\mathrm{p}^{*}(i \infty, 0)=[0,0,0,1]^{T}$ and obtain the idle-time probability $\Pi\left(\tau^{*}\right)$. We evaluate the detected residence time distribution as a conditioned distribution $\mathcal{W}_{r}\left(\tau^{*}\right)=-\partial_{\tau^{*}} \Pi\left(\tau^{*}\right)=p_{01}\left(\tau^{*}\right) \Gamma^{\mathrm{D}}$ and obtain

$$
\mathcal{W}_{r}\left(\tau^{*}\right)=\frac{2 \Gamma^{\mathrm{D}} \Gamma^{-}}{\Lambda} e^{-\tau^{*} \sum_{\alpha} \Gamma^{\alpha} / 2} \sinh \left[\tau^{*} \Lambda / 2\right],
$$

where $\Lambda=\sqrt{\left(\sum_{\alpha} \Gamma^{\alpha}\right)^{2}-4 \Gamma^{\mathrm{D}} \Gamma^{-}}$and $\alpha=+,-$, D. If we assume the detector to be perfect $\Gamma^{\mathrm{D}} \rightarrow \infty$, we recover the exponential decay of the occupied parasitic state $\mathcal{W}_{\infty}\left(\tau^{*}\right)=$ $\Gamma^{-} e^{-\tau^{*} \Gamma^{-}}$.

\section{EXPERIMENTAL SETUP}

We employ a superconducting charge detector which consists of two $\mathrm{Al} / \mathrm{Al}_{2} \mathrm{O}_{3} / \mathrm{Al}$ Josephson junctions in series with resistances and capacitances $R_{i}, C_{i}, i=1,2$ and a superconducting gap $\Delta$, which form a charge island with a charging energy of $E_{\mathrm{ch}}=e^{2} / C_{\Sigma}$, where $C_{\Sigma}=C_{1}+C_{2}+C_{\mathrm{c}}+C_{\mathrm{g}}, e$ is the elementary charge, $C_{\mathrm{g}}$ is the capacitive coupling between the gate and the island, and $C_{\mathrm{c}}$ is the mutual capacitance between the TLF and the detector island. The device is fabricated on a 500- $\mu$ m-thick high-resistivity silicon wafer with 5-nm-thick high-purity field oxide. The measurements are carried out in a cryostat that has a base temperature of $T=100 \mathrm{mK}$. Figure 1(b) shows the top view of a detector that is nominally identical to the device that is used to carry out the presented experiments. The $\mathrm{Al}$ leads provide galvanic contacts between the tunnel junctions, and the gate voltage $V_{\mathrm{g}}$ is used to tune the charge sensitivity of the SSET. The bias voltage $V_{\mathrm{dc}}$ controls the Fermi level of the lead, whereas the other lead is connected to a room temperature transimpedance amplifier. At the output of the amplifier, we measure the voltage and transmit the signal to a proportional-integral-derivative (PID) feedback circuit that maintains the gate charge point, and hence the charge sensitivity of the detector.

The charge stability of the detector, shown in Fig. 1(c), reveals how to associate the measured dc current with the gate-tunable charge point of the superconducting island. To minimize the detector backaction on the two-level fluctuator, we choose the bias voltage that corresponds to the double Josephson-quasiparticle process [63,64].

\section{RESULTS}

The signal produced by the TLF is read out using the detector such that the PID controller is utilized and tuned to a set point indicated in Fig. 1(d). In addition to the white noise, we observe a systematic signal, as exemplified in Fig. 2(a). The jumps are distinguished from the white noise when the amplitude of the detector signal exceeds the $5 \sigma$ white-noise level. Furthermore, the detector signal is filtered with a twopoint moving averaging window, which results in a detector bandwidth of $\Gamma^{\mathrm{D}} /(2 \pi)=11.25 \mathrm{~Hz}$.

First, a jump with a negative sign appears and the detector current offset takes a negative value. Then, the PID steers back to the set point using the detector gate. After $\tau^{*}$ residence time, another jump appears, but always with the opposite, positive sign compared to the first jump. The consecutive jump occurs at $\tau$ counted from the previous negative jump. The probability distribution of the jump amplitudes, shown in Fig. 2(b), seems Gaussian; however, the mean values and variances are different for the different jump directions.
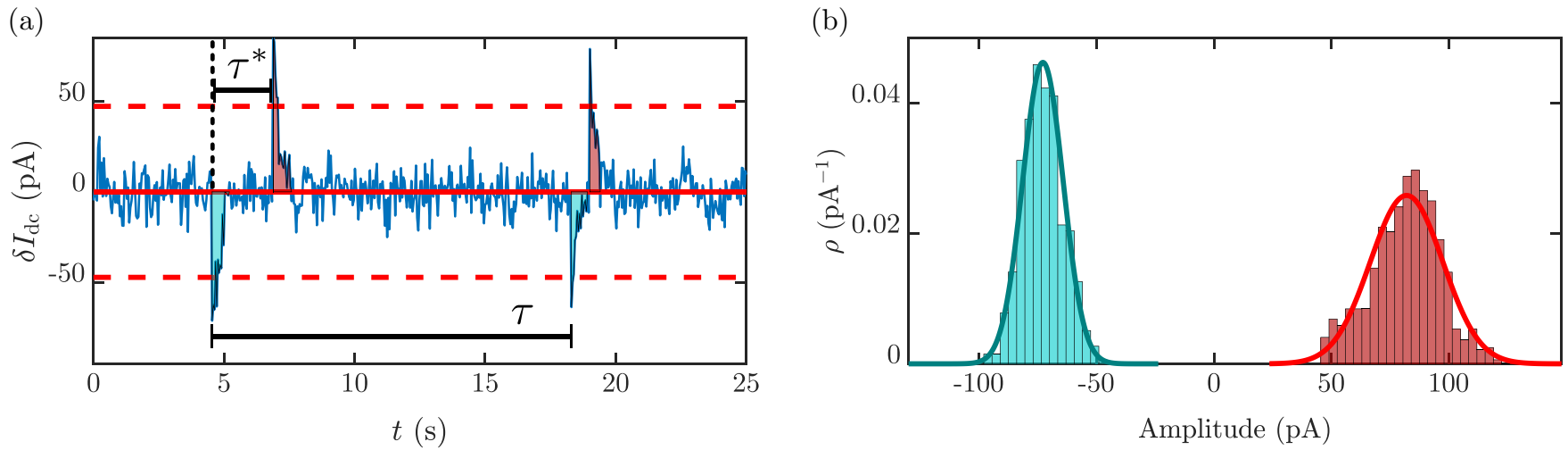

FIG. 2. (a) Electric current offset of the detector as a function of time (blue line). The two characteristic parameters, the waiting time $(\tau)$ and the residence time $\left(\tau^{*}\right)$ are indicated. The red dashed lines correspond to $\pm 5 \sigma$ white-noise levels and the red solid line depicts the PID set current, which is at the positive slope on the dashed line in Fig. 1(c). The shaded areas indicate the signal corresponding to the jump events. (b) Probability density of the charge signal, $\rho$, obtained by monitoring the jump amplitudes. The colors match with those of (a). 

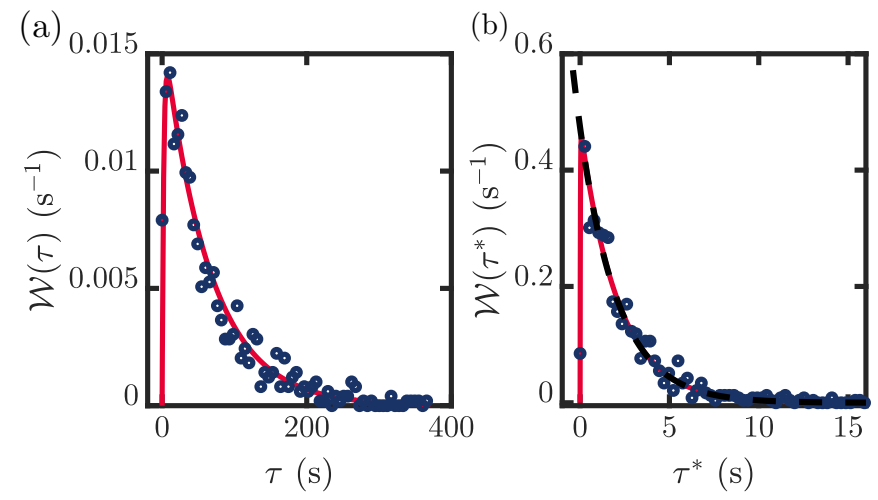

FIG. 3. (a) Measured (circles) and fitted (line) waiting time distribution of the charge jumps according to Eq. (7). (b) Measured (circles) and fitted residence time distribution of the charge jumps in the case of finite detector bandwidth (line) and ideal detector (dashed line) according to Eq. (9). The data have been recorded as illustrated in Fig. 2(a).

Since the switching between the two states is associated with single-electron transitions, $\tau$ and $\tau^{*}$ can be extracted from traces such as that shown in Fig. 2(a). The total duration of the measured time trace is $16 \mathrm{~h}$, which provides approximately 1000 back-and-forth tunneling events, giving rise to the WTD shown in Fig. 3(a).

We obtain the parameters $\Gamma^{+}$and $\Gamma^{-}$by fitting Eq. (7) to the data in Fig. 3(a). The fitted waiting time distribution, where the tunneling rates are $\Gamma^{+}=15.8 \times 10^{-3} \mathrm{~s}^{-1}$ and $\Gamma^{-}=473.2 \times 10^{-3} \mathrm{~s}^{-1}$, agrees well with the measured distribution. The significant difference between $\Gamma^{+}$and $\Gamma^{-}$ originates from the energy splitting of the two charge states and from the feedback; for every switching event the detector gate induces a compensating electrostatic field that not only affects the operation of the SSET, but also tends to polarize the TLF towards the opposite state. Since the average residence time $\left\langle\tau^{*}\right\rangle$ is significantly shorter than $\langle\tau\rangle$ and comparable to $1 / \Gamma^{\mathrm{D}}$, we fit Eq. (9) to the residence time distribution, shown in Fig. 3(b), by first fixing $\Gamma^{+}$and $\Gamma^{-}$according to the WTD. Thus, the only fitting parameter in the model is $\Gamma^{\mathrm{D}}$, with the estimated $\Gamma^{\mathrm{D}} /(2 \pi)=10.4 \mathrm{~Hz}$ based on the fit. The fitted $\left\langle\Gamma^{\mathrm{D}}\right\rangle$ deviates roughly $7.6 \%$ from the nominal detector bandwidth.

From the measured state transitions we can also construct the counting statistics $P(n, t)$ used in Eq. (2), where $n$ denotes the number of switching events regardless of the direction. The limited number of total jumps requires a moving time window to achieve the FCS with different time window lengths. Therefore, consecutive time windows are shifted by $1 \mathrm{~s}$ and used in the average over the 16-h time trace. The Markovian single-electron tunneling in a dot coupled to a charge reservoir follows the Poisson distribution [65]. If we assume an effective tunneling rate $\Gamma^{\text {eff }}$, we can fit a Poisson distribution to the data shown in Fig. 4. The extracted effective tunneling rate is $\Gamma^{\text {eff }}=31.5 \times 10^{-3} \mathrm{~s}^{-1}$, which can be explained by the branching of jumps observed in Fig. 2(a). More precisely, we effectively observe two events with a rate of $\Gamma^{+}$, since after each in-tunneling event there is an out-tunneling event with a brief delay.

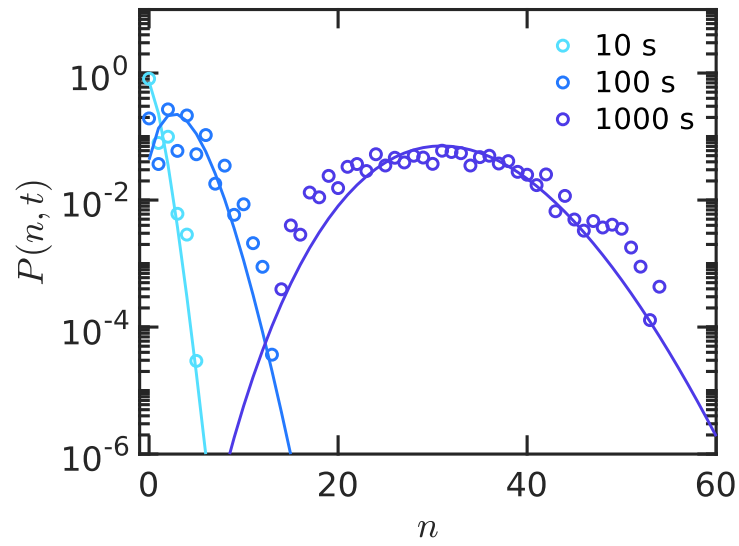

FIG. 4. Measured (circles) and fitted (lines) FCS of sequential single-electron tunneling events for three different time windows.

\section{CONCLUSIONS}

We have demonstrated that WTD is useful in extracting the time scales of a TLF. Including the finite bandwidth of the detector in the waiting time theory provides us with an accurate distribution of the waiting times in the charge trap. In contrast to WTD, full counting statistics covers long time scales and delivers mean values, noise, and high-order moments. Both approaches are powerful tools and they are connected [20]. From the observed probability distribution $P(n, t)$, we conclude that charge transitions in the TLF constitute a Poisson process. The distribution of waiting times, Eq. (7), indicates that there are two Poisson processes with different rates $\Gamma^{+}$and $\Gamma^{-}$in the two-level fluctuator. It requires less steps to evaluate the WTD than to evaluate the full counting statistics $P(n, t)$. Moreover, the waiting time formalism allows us to evaluate the WTD analytically where as an analytic expression for $P(n, t)$ is not currently available. Waiting times belong to the short-time-scale statistics and are sensitive to the finite detector bandwidth. Here, we have shown how the distribution of waiting times has obvious advantages in extracting the short time scales of the system.

\section{ACKNOWLEDGMENTS}

E.P. thanks C. Flindt for helpful comments. This work was carried out as part of the Academy of Finland Centre of Excellence program, Grants No. 312300, No. 308161, No. 314302, and No. 316551. E.P. acknowledges support from the Vilho, Yrjö and Kalle Väisälä Foundation of the Finnish Academy of Science and Letters through the grant for doctoral studies. We acknowledge the provision of facilities and technical support by Aalto University at OtaNano - Micronova and the NSW Node of the Australian National Fabrication Facility, where the devices were fabricated. We acknowledge funding from the Joint Research Project 'e-SI-Amp' (15SIB08) and the Australian Research Council (DP160104923). This project has received funding from the European Metrology Programme for Innovation and Research (EMPIR) co-financed by the Participating States and from the European Union Horizon 2020 research and innovation programme. 
[1] P. A. M. Holweg, J. Caro, A. H. Verbruggen, and S. Radelaar, Ballistic electron transport and two-level resistance fluctuations in noble-metal nanobridges, Phys. Rev. B 45, 9311 (1992).

[2] Yu. M. Galperin, Nanzhi Zou, and K. A. Chao, Resonant tunneling in the presence of a two-level fluctuator: Average transparency, Phys. Rev. B 49, 13728 (1994).

[3] Yu. M. Galperin and K. A. Chao, Resonant tunneling in the presence of a two-level fluctuator: Low-frequency noise, Phys. Rev. B 52, 12126 (1995).

[4] R. J. P. Keijsers, O. I. Shklyarevskii, and H. van Kempen, Point-Contact Study of Fast and Slow Two-Level Fluctuators in Metallic Glasses, Phys. Rev. Lett. 77, 3411 (1996).

[5] O. P. Balkashin, R. J. P. Keijsers, H. van Kempen, Yu. A. Kolesnichenko, and O. I. Shklyarevskii, Relaxation of two-level fluctuators in point contacts, Phys. Rev. B 58, 1294 (1998).

[6] S. Oh, K. Cicak, J. S. Kline, M. A. Sillanpää, K. D. Osborn, J. D. Whittaker, R. W. Simmonds, and D. P. Pappas, Elimination of two level fluctuators in superconducting quantum bits by an epitaxial tunnel barrier, Phys. Rev. B 74, 100502(R) (2006).

[7] J. Schriefl, Y. Makhlin, A. Shnirman, and G. Schön, Decoherence from ensembles of two-level fluctuators, N. J. Phys. 8, 1 (2006).

[8] N. M. Zimmerman, W. H. Huber, B. Simonds, E. Hourdakis, A. Fujiwara, Y.i Ono, Y. Takahashi, H. Inokawa, M. Furlan, and M. W. Keller, Why the long-term charge offset drift in Si singleelectron tunneling transistors is much smaller (better) than in metal-based ones: Two-level fluctuator stability, J. Appl. Phys. 104, 033710 (2008)

[9] J. Burnett, L. Faoro, I. Wisby, V. L. Gurtovoi, A. V. Chernykh, G. M. Mikhailov, V. A. Tulin, R. Shaikhaidarov, V. Antonov, P. J. Meeson, A. Ya Tzalenchuk, and T. Lindström, Evidence for interacting two-level systems from the $1 / \mathrm{f}$ noise of a superconducting resonator, Nat. Commun. 5, 4119 (2014).

[10] M. Möttönen, R. de Sousa, J. Zhang, and K. B. Whaley, Highfidelity one-qubit operations under random telegraph noise, Phys. Rev. A 73, 022332 (2006).

[11] L.-C. Ku and C. C. Yu, Decoherence of a Josephson qubit due to coupling to two-level systems, Phys. Rev. B 72, 024526 (2005).

[12] C. Müller, A. Shnirman, and Y. Makhlin, Relaxation of Josephson qubits due to strong coupling to two-level systems, Phys. Rev. B 80, 134517 (2009).

[13] J. Goetz, F. Deppe, M. Haeberlein, F. Wulschner, C. W. Zollitsch, Sebastian Meier, M. Fischer, P. Eder, E. Xie, K. G. Fedorov, E. P. Menzel, A. Marx, and R. Gross, Loss mechanisms in superconducting thin film microwave resonators, J. Appl. Phys. 119, 015304 (2016).

[14] S. P. Giblin, P. See, A. Petrie, T. J. B. M. Janssen, I. Farrer, J. P. Griffiths, G. A. C. Jones, D. A. Ritchie, and M. Kataoka, High-resolution error detection in the capture process of a single-electron pump, Appl. Phys. Lett. 108, 023502 (2016).

[15] M. Jenei, R. Zhao, K. Y. Tan, T. Tanttu, K. W. Chan, Y. Sun, V. Sevriuk, F. Hudson, A. Rossi, A. Dzurak, and M. Möttönen, Superconducting charge sensor coupled to an electron layer in silicon, arXiv:1909.11976.

[16] N. M. Zimmerman, J. L. Cobb, and A. F. Clark, Modulation of the charge of a single-electron transistor by distant defects, Phys. Rev. B 56, 7675 (1997).

[17] M. Furlan and S. V. Lotkhov, Electrometry on charge traps with a single-electron transistor, Phys. Rev. B 67, 205313 (2003).
[18] L. Sun and B. E. Kane, Detection of a single-charge defect in a metal-oxide-semiconductor structure using vertically coupled $\mathrm{Al}$ and Si single-electron transistors, Phys. Rev. B 80, 153310 (2009).

[19] A. Pourkabirian, M. V. Gustafsson, G. Johansson, J. Clarke, and P. Delsing, Nonequilibrium Probing of Two-Level Charge Fluctuators Using the Step Response of a Single-Electron Transistor, Phys. Rev. Lett. 113, 256801 (2014).

[20] T. Brandes, Waiting times and noise in single particle transport, Ann. Phys. 17, 477 (2008).

[21] S. Welack, M. Esposito, U. Harbola, and S. Mukamel, Interference effects in the counting statistics of electron transfers through a double quantum dot, Phys. Rev. B 77, 195315 (2008).

[22] S. Welack, S. Mukamel, and Y. J. Yan, Waiting time distributions of electron transfers through quantum dot AharonovBohm interferometers, Europhys. Lett. 85, 57008 (2009).

[23] S. Welack and Y. J. Yan, Non-Markovian theory for the waiting time distributions of single electron transfers, J. Chem. Phys. 131, 114111 (2009).

[24] K. H. Thomas and C. Flindt, Electron waiting times in nonMarkovian quantum transport, Phys. Rev. B 87, 121405(R) (2013).

[25] G.-M. Tang, F. Xu, and J. Wang, Waiting time distribution of quantum electronic transport in the transient regime, Phys. Rev. B 89, 205310 (2014).

[26] G.-M. Tang and J. Wang, Full-counting statistics of charge and spin transport in the transient regime: A nonequilibrium Green's function approach, Phys. Rev. B 90, 195422 (2014).

[27] B. Sothmann, Electronic waiting-time distribution of a quantum-dot spin valve, Phys. Rev. B 90, 155315 (2014).

[28] V. Talbo, J. Mateos, S. Retailleau, P. Dollfus, and T. González, Time-dependent shot noise in multi-level quantum dot-based single-electron devices, Semicond. Sci. Technol. 30, 055002 (2015).

[29] S. L. Rudge and D. S. Kosov, Distribution of residence times as a marker to distinguish different pathways for quantum transport, Phys. Rev. E 94, 042134 (2016).

[30] S. L. Rudge and D. S. Kosov, Distribution of tunnelling times for quantum electron transport, J. Chem. Phys. 144, 124105 (2016).

[31] K. Ptaszyński, Nonrenewal statistics in transport through quantum dots, Phys. Rev. B 95, 045306 (2017).

[32] S. L. Rudge and D. S. Kosov, Distribution of waiting times between electron cotunneling events, Phys. Rev. B 98, 245402 (2018).

[33] P. Stegmann, J. König, and S. Weiss, Coherent dynamics in stochastic systems revealed by full counting statistics, Phys. Rev. B 98, 035409 (2018).

[34] E. Kleinherbers, P Stegmann, and P König, Revealing attractive electron-electron interaction in a quantum dot by full counting statistics, New J. Phys. 20, 073023 (2018).

[35] G. Tang, F. Xu, S. Mi, and J. Wang, Spin-resolved electron waiting times in a quantum-dot spin valve, Phys. Rev. B 97, 165407 (2018)

[36] S. L. Rudge and D. S. Kosov, Nonrenewal statistics in quantum transport from the perspective of first-passage and waiting time distributions, Phys. Rev. B 99, 115426 (2019).

[37] M. Albert, G. Haack, C. Flindt, and M. Büttiker, Electron Waiting Times in Mesoscopic Conductors, Phys. Rev. Lett. 108, 186806 (2012). 
[38] G. Haack, M. Albert, and C. Flindt, Distributions of electron waiting times in quantum-coherent conductors, Phys. Rev. B 90, 205429 (2014).

[39] R. Seoane Souto, R. Avriller, R. C. Monreal, A. Martín-Rodero, and A. Levy Yeyati, Transient dynamics and waiting time distribution of molecular junctions in the polaronic regime, Phys. Rev. B 92, 125435 (2015).

[40] D. S. Kosov, Waiting time between charging and discharging processes in molecular junctions, J. Chem. Phys. 149, 164105 (2018).

[41] L. Rajabi, C. Pöltl, and M. Governale, Waiting Time Distributions for the Transport through a Quantum-Dot Tunnel Coupled to One Normal and One Superconducting Lead, Phys. Rev. Lett. 111, 067002 (2013).

[42] S. Dambach, B. Kubala, V. Gramich, and J. Ankerhold, Timeresolved statistics of nonclassical light in Josephson photonics, Phys. Rev. B 92, 054508 (2015).

[43] S. Dambach, B. Kubala, and J. Ankerhold, Time-resolved statistics of photon pairs in two-cavity Josephson photonics, Fortschr. Phys. 651600061 (2016).

[44] M. Albert, D. Chevallier, and P. Devillard, Waiting times of entangled electrons in normal-superconducting junctions, Physica E 76, 209 (2016).

[45] D. Chevallier, M. Albert, and P. Devillard, Probing Majorana and Andreev bound states with waiting times, Europhys. Lett. 116, 27005 (2016).

[46] N. Walldorf, C. Padurariu, A.-P. Jauho, and C. Flindt, Electron Waiting Times of a Cooper Pair Splitter, Phys. Rev. Lett. 120, 087701 (2018).

[47] S. Mi, P. Burset, and C. Flindt, Electron waiting times in hybrid junctions with topological superconductors, Sci. Rep. 8, 16828 (2018).

[48] D. Dasenbrook, P. P. Hofer, and C. Flindt, Electron waiting times in coherent conductors are correlated, Phys. Rev. B 91, 195420 (2015).

[49] F. Brange, P. Menczel, and C. Flindt, Photon counting statistics of a microwave cavity, Phys. Rev. B 99, 085418 (2019).

[50] M. Albert, C. Flindt, and M. Büttiker, Distributions of Waiting Times of Dynamic Single-Electron Emitters, Phys. Rev. Lett. 107, 086805 (2011).

[51] D. Dasenbrook, C. Flindt, and M. Büttiker, Floquet Theory of Electron Waiting Times in Quantum-Coherent Conductors, Phys. Rev. Lett. 112, 146801 (2014).

[52] E. Potanina and C. Flindt, Electron waiting times of a periodically driven single-electron turnstile, Phys. Rev. B 96, 045420 (2017).
[53] P. Burset, J. Kotilahti, M. Moskalets, and C. Flindt, Time-domain spectroscopy of mesoscopic conductors using voltage pulses, Adv. Quantum Technol. 2, 1970023 (2019).

[54] S. K. Gorman, Y. He, M. G. House, J. G. Keizer, D. Keith, L. Fricke, S. J. Hile, M. A. Broome, and M. Y. Simmons, Tunneling Statistics for Analysis of Spin-Readout Fidelity, Phys. Rev. Appl. 8, 034019 (2017).

[55] D. A. Bagrets and Yu. V. Nazarov, Full counting statistics of charge transfer in Coulomb blockade systems, Phys. Rev. B 67, 085316 (2003).

[56] C. Flindt, T. Novotný, A. Braggio, M. Sassetti, and A.-P. Jauho, Counting Statistics of Non-Markovian Quantum Stochastic Processes, Phys. Rev. Lett. 100, 150601 (2008).

[57] C. Flindt, T. Novotný, A. Braggio, and A.-P. Jauho, Counting statistics of transport through Coulomb blockade nanostructures: High-order cumulants and non-Markovian effects, Phys. Rev. B 82, 155407 (2010).

[58] M. Benito, M. Niklas, and S. Kohler, Full-counting statistics of time-dependent conductors, Phys. Rev. B 94, 195433 (2016).

[59] P. Talkner, Ł. Machura, M. Schindler, P. Hänggi, and J. Łuczka, Statistics of transition times, phase diffusion and synchronization in periodically driven bistable systems, New J. Phys. 7, 14 (2005).

[60] O. Naaman and J. Aumentado, Poisson Transition Rates from Time-Domain Measurements with a Finite Bandwidth, Phys. Rev. Lett. 96, 100201 (2006).

[61] S. Gustavsson, R. Leturcq, T. Ihn, K. Ensslin, M. Reinwald, and W. Wegscheider, Measurements of higher-order noise correlations in a quantum dot with a finite bandwidth detector, Phys. Rev. B 75, 075314 (2007).

[62] C. Flindt, C. Fricke, F. Hohls, T. Novotny, K. Netocny, T. Brandes, and R. J. Haug, Universal oscillations in counting statistics, Proc. Natl. Acad. Sci. USA 106, 10116 (2009).

[63] A. A. Clerk, S. M. Girvin, A. K. Nguyen, and A. D. Stone, Resonant Cooper-Pair Tunneling: Quantum Noise and Measurement Characteristics, Phys. Rev. Lett. 89, 176804 (2002).

[64] W. W. Xue, Z. Ji, F. Pan, J. Stettenheim, M. P. Blencowe, and A. J. Rimberg, Measurement of quantum noise in a singleelectron transistor near the quantum limit, Nat. Phys. 5, 660 (2009).

[65] V. F. Maisi, D. Kambly, C. Flindt, and J. P. Pekola, Full Counting Statistics of Andreev Tunneling, Phys. Rev. Lett. 112, 036801 (2014). 